\title{
Données nouvelles sur quelques espèces minérales de Haut-le-Wastia (Province de Namur, Belgique)
}

Manuscrit reçu le 10 février 2017 et accepté le 18 avril 2017

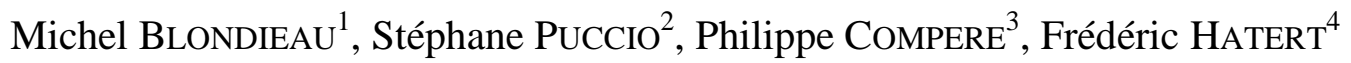

\author{
${ }^{1}$ Val des Cloches 131, B-6927 Tellin, Belgique \\ ${ }^{2}$ Rue des Fontaines 156, B-4041 Vottem, Belgique \\ ${ }^{3}$ Université de Liège, Département de Biologie, Écologie, Évolution, unité FOCUS, Cellule \\ d'Appui à la Recherche et à l'Enseignement en Microscopie (CAREM-ULg), B6c, B-4000 \\ Liège, Belgique \\ ${ }^{4}$ Université de Liège, Laboratoire de Minéralogie B18, B-4000 Liège, Belgique
}

\section{Résumé}

La béraunite, la fluorapatite et le xénotime-(Y) sont trois espèces nouvelles observées dans l'indice phosphaté de Haut-le-Wastia dont la minéralogie avait été initialement décrite en 1985 par R. Van Tassel. La présence de xénotime-(Y) en inclusion dans la fluorapatite et contenant un peu d'uranium, est une donnée nouvelle à considérer pour rendre compte de la radioactivité observée dans les couches de passage Viséen-Namurien du bassin de Dinant.

Mots clés : Haut-le-Wastia, minéraux, Namurien, Belgique, xénotime-(Y), radioactivité.

\begin{abstract}
Beraunite, fluorapatite and xenotime-(Y) are three new species for the phosphate spot in Haut-le-Wastia. A small crystal of Xenotime-(Y) included in fluorapatite is observed with some amounts of uranium and it's a new data to understand the radioactivity of the ViseanNamurian shales in the Dinant margin.
\end{abstract}

Keywords : Haut-le Wastia, minerals, Namurian, Belgium, xenotime-(Y), radioactive shales.

\section{Introduction}

Des travaux de rectification de la route Anhée-Haut-le-Wastia, réalisés en 1976-1977, ont recoupé des minéralisations phosphatées à proximité du village de Haut-le-Wastia. L'examen minéralogique de la tranchée, réalisé par Van Tassel (1985), a permis l'identification de nombreux phosphates dont la vashegyite, espèce nouvelle pour la Belgique. Plus tard, Dillen \& Van Goethem (1990) ont complété l'inventaire minéralogique du site en décrivant la ferristrunzite. Un nouvel examen d'échantillons collectés au début des années '90 nous a 
Bulletin de la Société Royale des Sciences de Liège, Vol. 86, articles, 2017, p. 49 - 56

permis d'étoffer encore cet inventaire ; le présent article fournit la description détaillée de ces minéraux.

\section{Contexte géologique}

Les roches renfermant les minéralisations de phosphates sont localisées dans les couches de passage du Viséen (calcaire) au Namurien (détritique) (V3c), observées dans la partie nord du bassin de Dinant. Charlet et al. (1983) et Ndziba (1982) ont remarqué la présence d'une anomalie radioactive de la coupe de Haut-le-Wastia avec des pics radioactifs localisés dans les couches de schistes riches en nodules phosphatés. D'autres anomalies radioactives ont été observées dans la région toujours dans ces mêmes couches de transition (V3c) riches en nodules phosphatés. Pour ces auteurs, l'anomalie radioactive au contact Viséen-Namurien présente donc un caractère stratiforme et continu à l'échelle du bassin. La présence d'espèces minérales bien exprimées n'est toutefois généralement pas observée dans ces différentes occurrences.

À Haut-le-Wastia, les minéralisations se rencontrent dans une zone dont la lecture géologique est compliquée. Voici la description qu'en donne Van Tassel (1985) : "... zone brouillée sur une vingtaine de mètres comprenant des schistes foncés zonaires, parfois entrelardés de straticules clairs, et des roches dures, siliceuses, parfois alvéolaires, puis de schistes grisbrun à lits de nodules phosphoriques, de roches schisteuses zonaires parfois fortement plissées ou broyées (brèches tectonique), de schistes noirs et de roches grises siliceuses alvéolaires."

\section{Descriptions de trois nouvelles espèces minérales à Haut-le-Wastia.}

Les minéraux ont été soumis à une analyse par diffraction des rayons $\mathrm{X}$ en mode «DebyeScherrer » sur un diffractomètre à 4 cercles Rigaku Xcalibur, équipé d'un détecteur EOS et utilisant la radiation $\operatorname{MoK}_{\alpha}(\lambda=0,7107 \AA)$. La morphologie des minéraux a été observée à l'aide d'un microscope électronique à balayage environnemental FEI ESEM-FEG XL 30 (CAREM-ULg, Cellule d'Appui à la Recherche et à l'Enseignement en Microscopie de l'Université de Liège). Les images ont été obtenues en électrons secondaires (mode «hautvide ») sous une tension d'accélération de $15 \mathrm{kV}$ et sur des échantillons métallisés au platine. Des spectres EDS («Energy Dispersive X-ray Spectrometry ») de microanalyse élémentaire aux rayons $\mathrm{X}$ ont été obtenus avec le même microscope, équipé d'un détecteur de rayons $\mathrm{X}$ 
Bulletin de la Société Royale des Sciences de Liège, Vol. 86, articles, 2017, p. 49 - 56

Si(Li) EDAX ou SDD Bruker $129 \mathrm{eV}$. Selon les cas, la tension d'accélération était réglée à 15, 20 ou $30 \mathrm{kV}$. Les spectres ont été traités soit par le logiciel EDAX, soit par le logiciel Quanta Esprit 1.9 qui a fourni l'identification des éléments et des analyses semi-quantitatives basées sur la matrice de correction ZAF sans standard.

Les descriptions suivantes concernent seulement les minéraux non signalés précédemment. Ils sont décrits par ordre alphabétique, et leur formule chimique idéale sont tirées de la liste officielle de la CNMNC-IMA (2016).

\subsection{Béraunite, $\mathrm{Fe}^{2+} \mathrm{Fe}^{3+}{ }_{5}\left(\mathrm{PO}_{4}\right)_{4}(\mathrm{OH})_{5} .6 \mathrm{H}_{2} \mathrm{O}$}

Le minéral se présente en petites plages de couleur vert foncé ou brun rouge en association avec de la ferristrunzite dans des blocs bréchiques brunâtres. L'analyse par diffraction des rayons $\mathrm{X}$ indique qu'il s'agit d'un minéral du groupe de la béraunite, ce que confirme l'analyse chimique qualitative EDS qui montre les seuls éléments $\mathrm{O}$, Fe et $\mathrm{P}$.

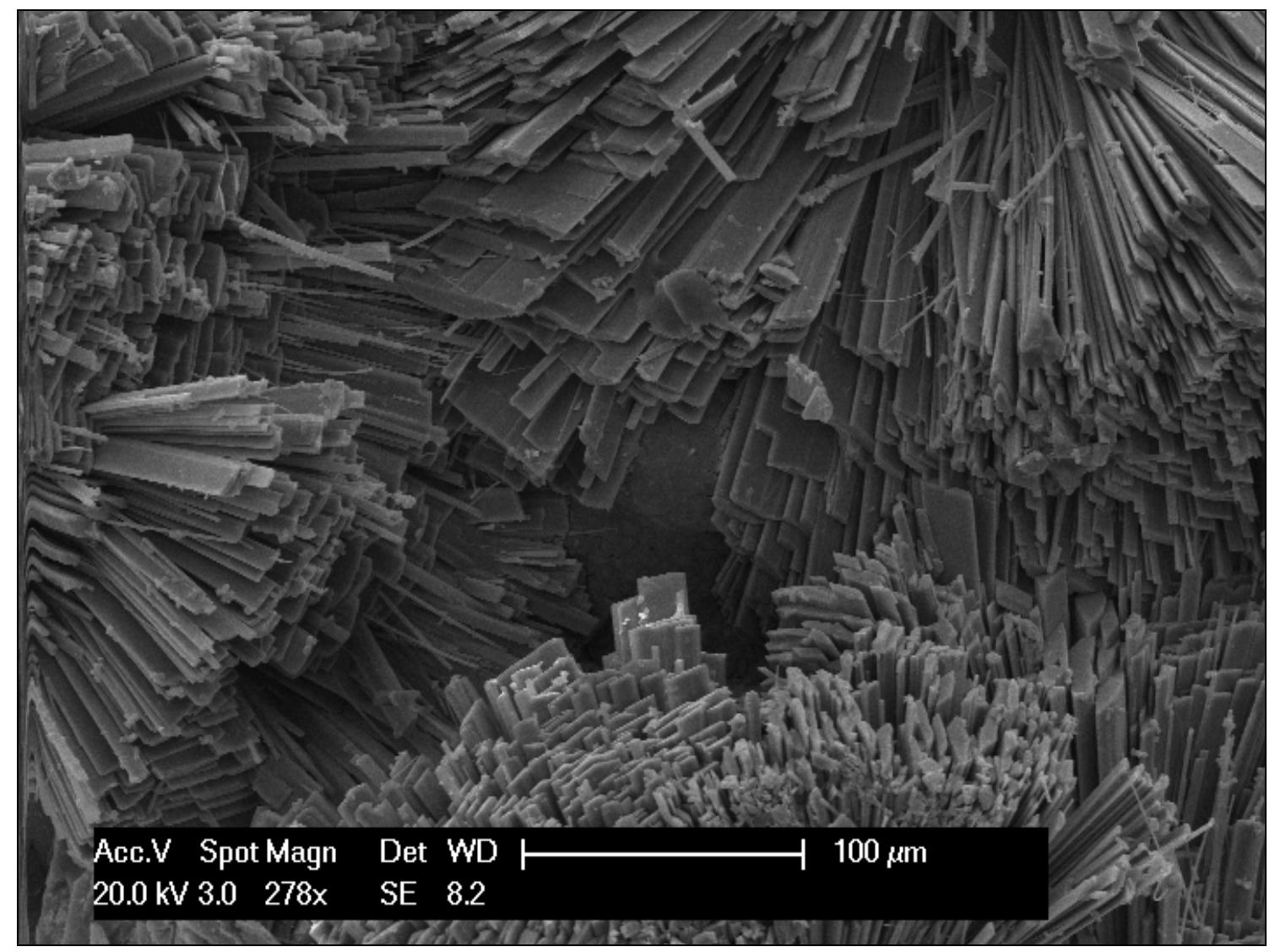

Fig. 1 : Cristaux de béraunite (Échantillon HLW-1, photographie au microscope électronique à balayage, mode électrons secondaires). 
En Belgique, la béraunite a été décrite à Blaton et à Richelle (Hatert et al., 2002). Comme à Blaton, la béraunite de Haut-le-Wastia est observée en association avec la ferristrunzite et se présente notamment en rosettes constituées de microcristaux lamellaires brillants vert foncé ou brun rouge. L'aspect des échantillons de Blaton, observés en microscopie électronique (Van Tassel \& Wouters, 1984), est fort semblable à ceux de Haut-le-Wastia

\subsection{Fluorapatite, $\mathrm{Ca}_{5}\left(\mathrm{PO}_{4}\right)_{3} \mathrm{~F}$}

Des cristaux en tonnelets hexagonaux blancs millimétriques, d'aspect terreux, ont été repérés par observation à la loupe binoculaire. Une analyse par diffraction des rayons $\mathrm{X}$ indique qu'il s'agit d'un minéral du groupe de l'apatite et l'analyse chimique qualitative par EDS montre la présence des éléments $\mathrm{O}, \mathrm{Ca}, \mathrm{P}$ et $\mathrm{F}$ ainsi que $\mathrm{Al}$ et $\mathrm{Fe}$ très minoritaires. Ces résultats indiquent qu'il s'agit de fluorapatite.

Ces cristaux sont cependant d'une très grande fragilité et se brisent rapidement quand on tente de les extraire. Les photos en microscopie électronique révèlent une structure très poreuse voire squelettique, rendant compte de leur extrême fragilité. Les cristaux initiaux ont subi des attaques chimiques qui les ont dissous presque entièrement, et il ne reste plus que quelques piliers rappelant vaguement la charpente cristalline originelle.

La fluorapatite n'était pas signalée par Hatert et al. (2002) à Haut-le-Wastia. Par contre, les roches où ont été observées les minéralisations sont connues pour leur teneur importante en phosphore. Van Tassel (1985) signale : "... apatite s.l. se présentant comme élément pétrographique dans des nodules centimétriques alignés selon la stratification...". Ndziba (1982) a également indiqué qu'il s'agissait d'apatite et non de "carbonate-apatite" sur base des diffractogrammes de poudres de rayons X. La "carbonate-apatite" n'est plus une espèce valide mais une variété de fluorapatite ou d'hydroxylapatite. 
Bulletin de la Société Royale des Sciences de Liège, Vol. 86, articles, 2017, p. 49 - 56

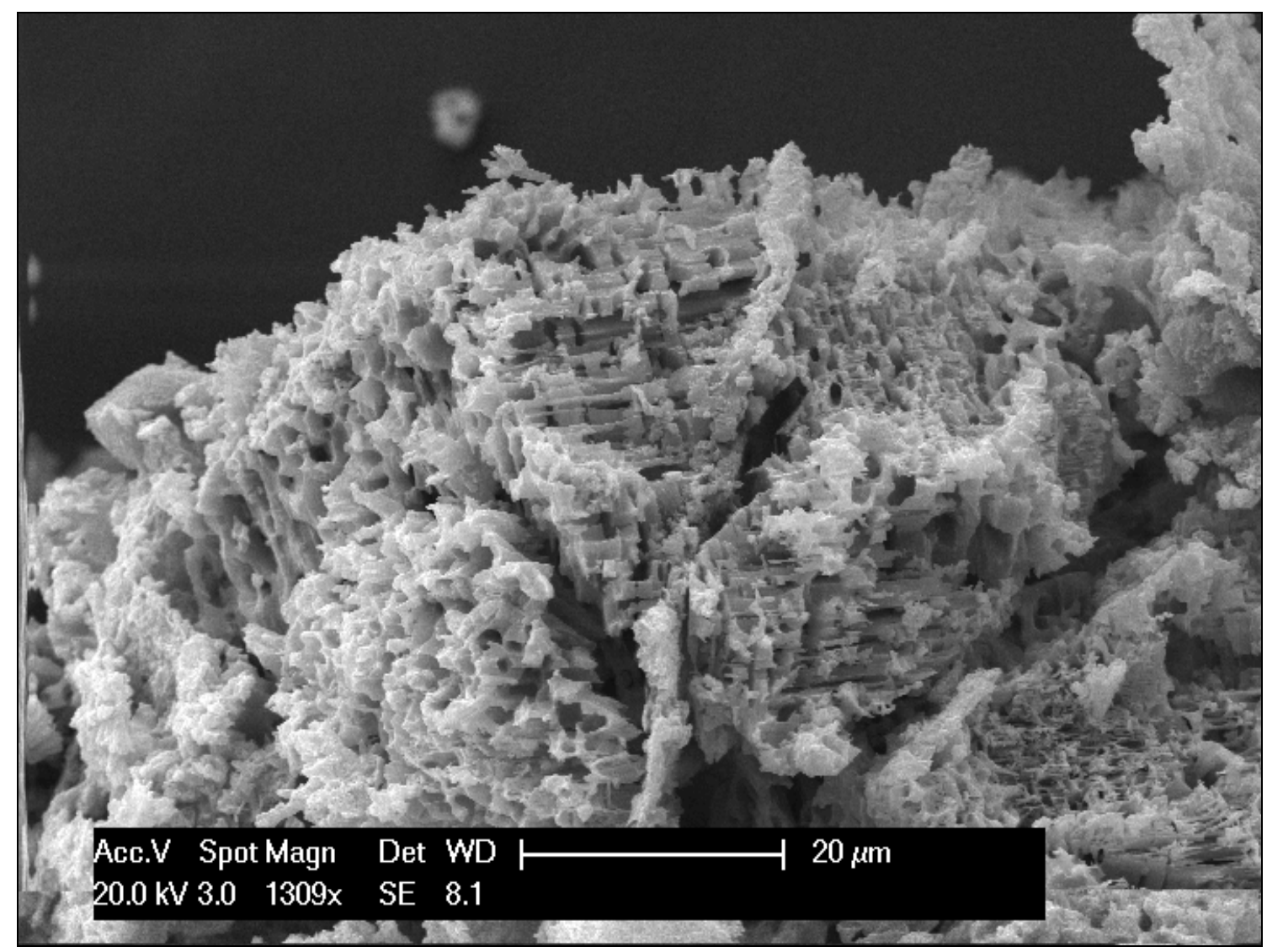

Fig. 2 : Fluorapatite squelettique (Échantillon HLW-2, photographie au microscope électronique à balayage, mode électrons secondaires).

\subsection{Xénotime-(Y), $\mathrm{YPO}_{4}$}

L'examen, en microscopie électronique, de cristaux de fluorapatite réduits en poussière du fait de leur grande fragilité, a permis la découverte de petits cristaux initialement inclus dans l'apatite. Un de ces cristaux, d'une vingtaine de microns, montre un habitus quadratique surmonté d'une pyramide (Figure 3). L'analyse chimique qualitative de ce cristal met en évidence trois éléments principaux : l'oxygène, le phosphore et l'yttrium. L'analyse chimique semi-quantitative indique le phosphore et l'yttrium en mêmes quantités stoechiométriques et permet d'assimiler ce cristal au xénotime-(Y). D'autres éléments minoritaires sont également présents : $\mathrm{Si}, \mathrm{Fe}, \mathrm{Ca}$ et $\mathrm{Al}$, ainsi que quelques éléments de terres rares ( $\mathrm{Er}, \mathrm{Yb}, \mathrm{Gd})$ et l'uranium. Il s'agit donc effectivement de xénotime-(Y). 
Le xénotime-(Y) est une espèce pouvant renfermer de l'erbium et d'autres lanthanides. L'uranium et le calcium peuvent aussi se substituer à l'yttrium, ce qui est le cas pour le xénotime-(Y) de Haut-le-Wastia. En Belgique, le xénotime-(Y) est déjà décrit à Nil-SaintVincent, dans l'indice radioactif de Daverdisse (Hatert et al., 2002), ainsi qu'à Salmchâteau (Blondieau et al., 2017).

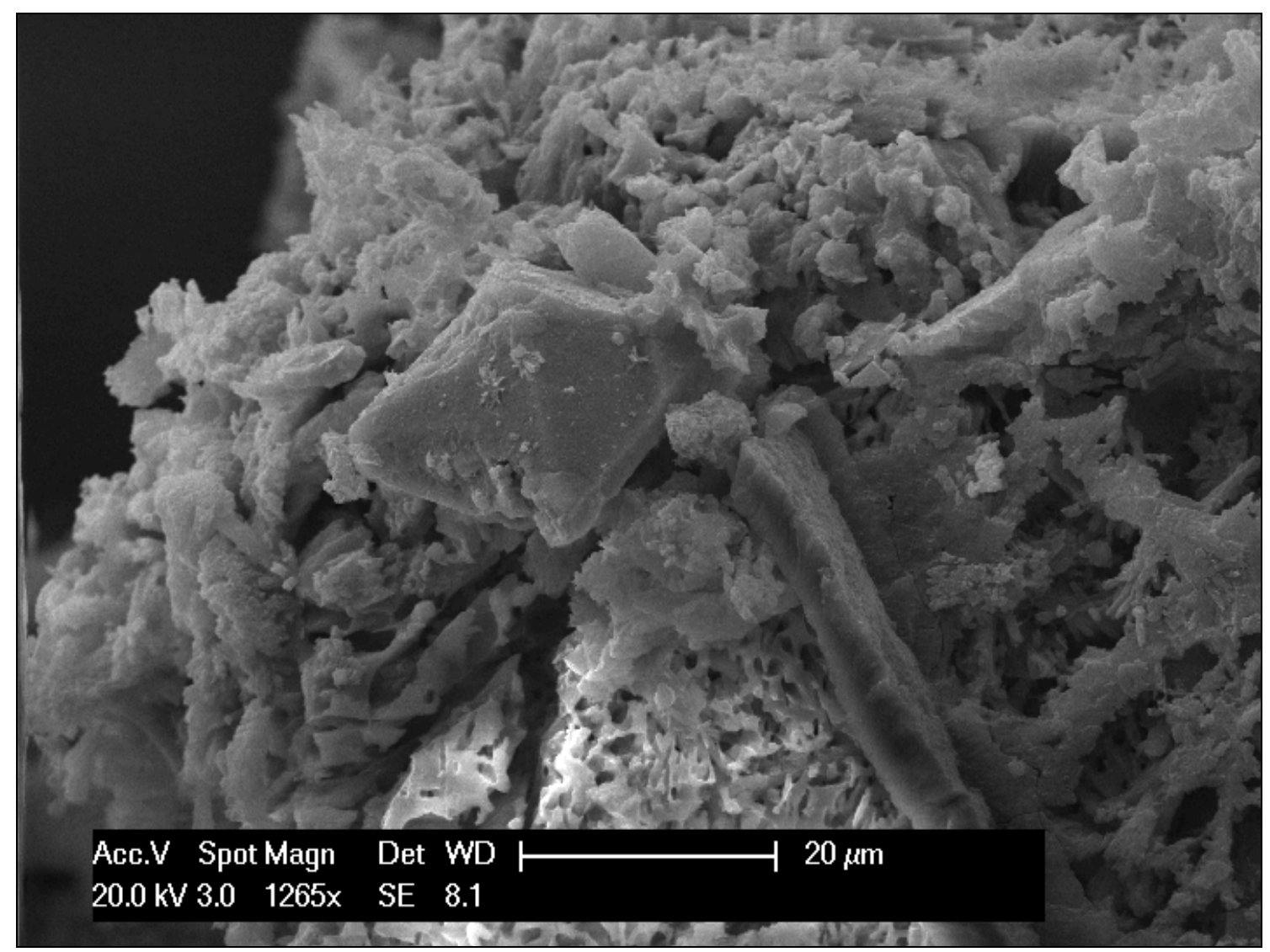

Fig. 3 : Xénotime-(Y) associé à de la fluorapatite squelettique (Échantillon HLW-2, photographie au microscope électronique à balayage, mode électrons secondaires).

\section{Conclusions}

La béraunite de Haut-le-Wastia est la troisième occurrence belge de ce minéral; les deux autres étant Blaton et Richelle.

Le xenotime-(Y) décrit dans cette note est une espèce porteuse d'un peu d'uranium, et la présence d'un petit cristal en inclusion dans la fluorapatite est une donnée nouvelle pour rendre compte de la radioactivité de ces couches de passage Viséen-Namurien. En effet, l'uranium n'a jusqu'à présent jamais été observé sous forme minéralogique dans ces anomalies radioactives du bassin de Dinant. Ndziba (1982) indique : "Ni l'étude en lames minces, ni 
Bulletin de la Société Royale des Sciences de Liège, Vol. 86, articles, 2017, p. 49 - 56

l'application de la diffraction des rayons $X$, ni les analyses autoradiographiques n'ont montré la présence des minéraux uranifères, bien définis dans la série étudiée; l'uranium est partout à l'état diffus.".

Le Tableau 1 ci-dessous reprend la liste complète des espèces minérales décrites à Hautle-Wastia.

Tableau 1: Espèces minérales recensées à Haut-le-Wastia. En gras, les espèces minéralogiques nouvelles pour la localité.

\begin{tabular}{|c|c|c|}
\hline Minéral & Formule chimique idéale & Références * \\
\hline Béraunite & $\mathrm{Fe}^{2+} \mathrm{Fe}^{3+}{ }_{5}\left(\mathrm{PO}_{4}\right)_{4}(\mathrm{OH})_{5} \cdot 6 \mathrm{H}_{2} \mathrm{O}$ & $(3)$ \\
\hline Cacoxénite & $\mathrm{Fe}^{3+}{ }_{24} \mathrm{AlO}_{6}\left(\mathrm{PO}_{4}\right)_{17}(\mathrm{OH})_{12 .} 75 \mathrm{H}_{2} \mathrm{O}$ & (1) \\
\hline Crandallite & $\mathrm{CaAl}_{3}\left(\mathrm{PO}_{4}\right)\left(\mathrm{PO}_{3} \mathrm{OH}\right)(\mathrm{OH})_{6}$ & (1) \\
\hline Destinézite & $\mathrm{Fe}^{3+}{ }_{2}\left(\mathrm{PO}_{4}\right)\left(\mathrm{SO}_{4}\right)(\mathrm{OH}) \cdot 6 \mathrm{H}_{2} \mathrm{O}$ & (1) \\
\hline Ferristrunzite & $\mathrm{Fe}^{3+} \mathrm{Fe}^{3+}{ }_{2}\left(\mathrm{PO}_{4}\right)_{2}(\mathrm{OH})_{3} .5 \mathrm{H}_{2} \mathrm{O}$ & (2) \\
\hline Fluorapatite & $\mathrm{Ca}_{5}\left(\mathrm{PO}_{4}\right)_{3} \mathrm{~F}$ & (3) \\
\hline Goethite & $\mathrm{FeO}(\mathrm{OH})$ & (1) \\
\hline Gypse & $\mathrm{Ca}\left(\mathrm{SO}_{4}\right) \cdot 2 \mathrm{H}_{2} \mathrm{O}$ & (1) \\
\hline Jarosite & $\mathrm{KFe}_{3}^{3+}\left(\mathrm{SO}_{4}\right)_{2}(\mathrm{OH})_{6}$ & (1) \\
\hline Natrojarosite & $\mathrm{NaFe}^{3+}{ }_{3}\left(\mathrm{SO}_{4}\right)_{2}(\mathrm{OH})_{6}$ & (1) \\
\hline Phosphosidérite & $\mathrm{Fe}^{3+}\left(\mathrm{PO}_{4}\right) \cdot 2 \mathrm{H}_{2} \mathrm{O}$ & (1) \\
\hline Quartz & $\mathrm{SiO}_{2}$ & (1) \\
\hline Sidérite & $\mathrm{Fe}\left(\mathrm{CO}_{3}\right)$ & (1) \\
\hline Vashegyite & $\mathrm{Al}_{11}\left(\mathrm{PO}_{4}\right)_{9}(\mathrm{OH})_{6} \cdot 38 \mathrm{H}_{2} \mathrm{O}$ & (1) \\
\hline Wavellite & $\mathrm{Al}_{3}\left(\mathrm{PO}_{4}\right)_{2}(\mathrm{OH})_{3} .5 \mathrm{H}_{2} \mathrm{O}$ & (1) \\
\hline Xénotime-(Y) & $\mathrm{YPO}_{4}$ & (3) \\
\hline
\end{tabular}

\section{Références}

Blondieau M., Puccio S., Compère P. \& Hatert F. (2017). Données nouvelles sur quelques espèces minérales de Vielsalm et de Salmchâteau (Province de Luxembourg, Belgique). Bulletin de la Société Royale des Sciences de Liège, 86, 1 - 48.

Charlet J.-M., Dejonghe L., De Witte, S.-M., Drumel B., Henry J., Herbosch A., Lefin J.-P. \& Martin H. (1983). Reconnaissance survey for uranium in the Belgian Paleozoic. Prof Paper, Service géologique de Belgique, 196, 189 pages.

Dillen R. \& Van Goethem L. (1990). Ferristrunzite - Korte mededelingen bijeengebracht door UMIBEL. Bulletin de la Société Belge de Géologie, 99/3-4, 399.

Hatert F., Deliens M., Fransolet A.-M. \& Van Der Meersche E. (2002). Les minéraux de Belgique, deuxième édition, 304 pages. 
Bulletin de la Société Royale des Sciences de Liège, Vol. 86, articles, 2017, p. 49 - 56

Ndziba M. (1982). Anomalies radiométriques des couches de passage du Viséen-Namurien dans le bassin de Dinant (Ardennes, Belgique). Etude géologique et géochimique. Thèse de doctorat $3^{e}$ cycle Univ. Lille, 153 pp. https://ori-nuxeo.univlille1.fr/nuxeo/site/esupversions/06fc13e2-9632-4b71-8e40-93d4c4666bdd

Van Tassel R. \& Wouters K. (1984). Mineralen bekeken met de elektronenmicroscoop Fosfaatmineralen van Blaton, provincie Henegouwen. Koninklijk Belgisch Instituut voor Natuurwetenschappen, 11 pages.

Van Tassel R. (1985). Minéraux phosphates secondaires (vashegyite, destinézite, wavellite, crandallite, phosphate de fer) à Haut-le-Wastia, province de Namur (Belgique). Bulletin de la Société Belge de Géologie, 94/1, 19-27. 